\title{
A polytopic strategy for improved non-asymptotic robust control via implicit Lyapunov functions
}

\author{
Alan Tapia ${ }^{\mathrm{a}, \mathrm{b}}$, Denis Efimov ${ }^{\mathrm{c}}$, Miguel Bernal ${ }^{\mathrm{b}}$, Leonid Fridman ${ }^{\mathrm{a}, *}$, Andrey \\ Polyakov $^{\mathrm{c}}$ \\ ${ }^{a}$ Department of Control and Robotics, Engineering Faculty, National Autonomous \\ University of Mexico, 04510, Mexico City, Mexico \\ ${ }^{b}$ Department of Electric and Electronics Engineering, Sonora Institute of Technology, \\ 85000, Cd. Obregon, Mexico \\ ${ }^{c}$ Inria, Univ. Lille, CNRS, UMR 9189 - CRIStAL, F-59000 Lille, France and ITMO \\ University, 49 Av. Kronverkskiy, 197101 Saint Petersburg, Russia
}

\begin{abstract}
This paper is concerned with finite- and fixed-time robust stabilization of uncertain multi-input nonlinear systems via the implicit Lyapunov function method. Instead of splitting the system into a linear nominal model and an additive perturbation which gathers nonlinearities, parametric uncertainties, and exogenous disturbances, the methodology hereby proposed preserves some nonlinear terms in the nominal system via an exact polytopic representation which leads to design conditions in the form of linear matrix inequalities. As a result, feasible solutions are found where former approaches fail; these solutions have more accurate settling-time estimates with reduced control effort. The corresponding control law includes well-known high-order sliding modes as a particular case. Numerical simulations are provided to illustrate the advantages of the proposal.
\end{abstract}

Keywords: Finite- and Fixed-Time Convergence, Implicit Lyapunov Functions, Homogeneity, Polytopic Systems, Linear Matrix Inequalities.

\footnotetext{
* Corresponding author

Email address: Ifridman@unam.mx (Leonid Fridman)
} 


\section{Introduction}

Robustness and convergence time are among the characteristics that describe the quality of a control law. Non-asymptotic stabilization (finite-time or fixed-time) has been achieved in a variety of ways [1]; among them, sliding mode control remains the most common solution by ensuring finite-time convergence of the system trajectories to a sliding manifold, even when the plant is in the presence of a certain class of uncertainties and disturbances [2]. Nevertheless, the chattering phenomenon limits the practical use of traditional schemes; then, higher-order sliding modes (HOSM) [3] were introduced as an attempt to alleviate this problem by substituting the discontinuous nature of the control law by a continuous one. However, the selection of control parameters to do so is not trivial and is still under study [4].

Originally, stability, robustness and convergence rate for HOSM algorithms were commonly analyzed by geometric [3] or homogeneous approaches [5]. Recall that homogeneity is a powerful tool for finite-time stability analysis since asymptotic stability of the origin of a homogeneous control system of negative degree implies global finite-time stability. Nevertheless, estimation of the settling time and tuning of control parameters cannot be achieved by those approaches. The main approach to solve this problem is based on the use of Lyapunov functions, mostly quadratic-like $[6,7,8]$. More recently, implicit Lyapunov functions (ILFs) $[9,10]$ have been adapted to the HOSM context [11]. This work follows the latter approach.

ILF-based control algorithms in $[12,13,14]$ have been proven to guarantee robust non-asymptotic stability for the nominal linear plant, where nonlinearities and uncertainties are considered as external disturbances. Yet, this consideration affects the control performance because there is a direct relationship between the size of the disturbances, on the one hand, and the restrictiveness of the stability conditions and the estimate of the settling time boundary, on the other hand. Based on this observation, our proposal to overcome this issue is based on polytopic representations.

Polytopic models were originally understood as belonging to the class of linear parameter varying (LPV) systems [15, 16]; later on, their use has been extended to nonlinear - possibly uncertain- models by means of exact convex rewriting of bounded nonlinearities and uncertainties, a methodology known as sector nonlinearity approach [17]. The resulting models are known as quasi-LPV when the interpolated systems are linear [18], or polynomial when they are of this nature [19]; both of them, when combined with the 
direct Lyapunov function method, lead to conditions in the form of linear matrix inequalities (LMIs) or sum of squares (SOS), which belong to the class of convex optimization problems [20]. Such formulation is highly appreciated in the control community as conditions, including those specifying performances, can be solved in polynomial time via commercially available software [21, 22]. Traditional sliding modes [23, 24, 25], second-order sliding sets [26, 27], and nonlinear sliding surface design for nonlinear systems [28] have already benefited from employing polytopic structures.

In this sense, the present work develops ILF-based finite- as well as fixedtime stabilization for multi-input uncertain nonlinear systems, without the necessity of considering nonlinearities as exogenous disturbances allowing us to obtain smaller settling time estimates and less restrictive stability conditions. Instead, the nonlinearities are incorporated in a nonlinear nominal system, which in turn is subsumed in a polytopic representation. HOSM algorithms can be obtained as a particular case of the proposed ILF-based control. Stability conditions are expressed in the form of LMIs, which allows tuning the control parameters via a convex optimization problem.

Motivation example: Consider the following second-order nonlinear system:

$$
\begin{aligned}
& \dot{x}_{1}=(\theta+0.5) x_{2}+2 \sin x_{2} \\
& \dot{x}_{2}=x_{1}^{2}+x_{2}+u+f_{m}(t, x),
\end{aligned}
$$

with $x_{1}, x_{2}$ are the scalar state variables, $\theta$ is a bounded parametric uncertainty $|\theta| \leq 0.2$ which can be time-varying, $f_{m}(t, x)$ is an unknown but bounded function $\left|f_{m}(t, x)\right| \leq 1$, and $u$ is the control input given by $u=$ $-x_{1}^{2}-x_{2}+v$, with $v$ to be designed.

The above model can be rewritten as follows

$$
\dot{x}=A x+B v+\underbrace{\left[\begin{array}{c}
(\theta-0.5) x_{2}+2 \sin x_{2} \\
f_{m}(t, x)
\end{array}\right]}_{d(t, x)}, \quad A=\left[\begin{array}{ll}
0 & 1 \\
0 & 0
\end{array}\right], B=\left[\begin{array}{l}
0 \\
1
\end{array}\right]
$$

where $x=\left[\begin{array}{ll}x_{1} & x_{2}\end{array}\right]^{T}$ and $d(t, x)=\left[\begin{array}{ll}d_{1}(t, x) & d_{2}(t, x)\end{array}\right]^{T}$ can be treated as an external disturbance with no distinction of its parametric, exogenous, or nonlinear nature.

To stabilize the origin of the latter system in a finite time, the following 
control law

$$
v=V^{1-\mu} K D\left(V^{-1}\right) x, \quad D\left(V^{-1}\right)=\left[\begin{array}{cc}
V^{-1-\mu} & 0 \\
0 & V^{-1}
\end{array}\right], \quad V^{-1}=\frac{1}{V}, \quad K \in \mathbb{R}^{1 \times 2}
$$

can be utilized (see, e.g. [13] for more details), where $\mu \in(0,1]$ and $V: \mathbb{R}^{2} \rightarrow$ $[0,+\infty)$ is a positive definite Lyapunov function $x \rightarrow V$ defined implicitly as a solution of the following non-linear equation

$$
x^{T} D\left(V^{-1}\right) P D\left(V^{-1}\right) x=1, \quad P=P^{T} \in \mathbb{R}^{2 \times 2} .
$$

The positive definite matrix $P$ can always be selected such that the latter equation has a unique positive definite solution $V(x)$, and the control (3) is smooth on $\mathbb{R}^{2} \backslash\{0\}$ and locally/globally (for $\mu=1$ ) bounded. In [13] the following LMI-based scheme for tuning of the control parameters $K=Y X^{-1}$ and $P=X^{-1}$ has been suggested

$$
A X+X A^{T}+B Y+Y^{T} B^{T}+\alpha X+\beta I_{2}<0, \quad X H_{\mu}+H_{\mu} X<0, \quad X>0,
$$

where $\alpha>\beta>0$ and $H_{\mu}=\operatorname{diag}\{-1-\mu,-1\}$. According to [13], the resulting control (3) stabilizes the origin of the system (2) provided that

$$
\beta^{2} \geq \frac{d_{1}^{2}(t, x)}{V^{2}(x)}+\frac{d_{2}^{2}(t, x)}{V^{2-2 \mu}(x)}
$$

The latter restriction is given a-posteriori (i.e. after a selection of control parameters) and describes a class of disturbances to be rejected.

Let us check if the condition (5) holds for the system (2). Since $d_{2}$ is assumed to be uniformly bounded and $V$ vanishes at zero then the inequality (5) may hold only for $\mu=1$. Notice that $\left|d_{1}\right|^{2} \leq\left(\theta-0.5+2 x_{2}^{-1} \sin x_{2}\right)^{2} x_{2}^{2}$ and the function $V$ is homogeneous [14], i.e. $V\left(\lambda^{2} x_{1}, \lambda x_{2}\right)=\lambda V\left(x_{1}, x_{2}\right), \forall \lambda>$ $0, \forall x \in \mathbb{R}^{2}$. Hence, we derive

$$
\frac{d_{1}^{2}(t, x)}{V^{2}(x)} \leq(1.7)^{2} \frac{x_{2}^{2}}{V^{2}(x)} \leq(1.7)^{2} \sup _{x: V(x)=1} x_{2}^{2} \leq(1.7)^{2} \sup _{x^{T} P x=1} x_{2}^{2} .
$$

Therefore, the inequality (5) with $\mu=1$ can be reduced (with a minor conservatism) to

$$
\beta^{2} \geq(1.7)^{2} \sup _{x^{T} P x=1} x_{2}^{2}+1
$$


The latter means that the selection of $P$ and $K$ using the above LMI is not sufficient for the finite-time stabilization of the system (2). The parameters $\beta$ and $P$ have to be selected such that the non-linear inequality (6) holds as well. In other words, the inequalities (6) and (4) have to be solved together in order to stabilize the system (2). However, it is not clear how to do this even in the planar case.

The main aim of the paper is to develop an LMI-based methodology of the ILF-control design allowing a-priori known restrictions on system perturbations/nonlinearities to be involved into control parameters tuning from the beginning. The key idea is to use an exact convex representation of bounded nonlinearities and uncertainties.

This paper is organized as follows: section 2 introduces the class of nonlinear systems under consideration, the methodology dealing with the convex modeling, definitions of finite- and fixed-time stability as well as a brief description of the employed ILF method; section 3 develops the main contribution, i.e., an ILF control design for robust finite- and fixed-time stabilization of uncertain nonlinear systems exactly represented as polytopic models altogether with a discussion on implementation issues; simulation results to point out the effectiveness of the proposed approach are shown in section 4; finally, section 5 draws some conclusions and remarks.

\section{Preliminaries}

\subsection{Convex modelling}

We consider the class of systems that can be described in the regular form:

$$
\begin{aligned}
& \dot{\eta}=a_{11}(\eta, \xi) \eta+a_{12}(\eta, \xi) \xi+d_{1}(t, \eta, \xi) \\
& \dot{\xi}=a_{21}(\eta, \xi) \eta+a_{22}(\eta, \xi) \xi+b(\eta, \xi) u+d_{2}(t, \eta, \xi),
\end{aligned}
$$

where $\eta \in \mathbb{R}^{n-m}$ and $\xi \in \mathbb{R}^{m}$ are components of the state, $u \in \mathbb{R}^{m}$ is the control input; $a_{i j}(\eta, \xi), i, j \in\{1,2\}$, and $b(\eta, \xi)$ are sufficiently smooth possibly nonlinear matrix expressions of adequate dimensions, $b(\eta, \xi)$ is nonsingular, and $d_{1}(t, \eta, \xi), d_{2}(t, \eta, \xi)$ are measurable essentially bounded time functions that describe exogenous disturbances and perturbations. For reasons that will become clear later, $a_{11}(\eta, \xi)$ will be considered as a perturbation in subsequent rearrangements.

Let us select the following control law

$$
u=(b(\eta, \xi))^{-1}\left(v(\eta, \xi)-\left[\begin{array}{ll}
a_{21}(\eta, \xi) & a_{22}(\eta, \xi)
\end{array}\right]\left[\begin{array}{l}
\eta \\
\xi
\end{array}\right]\right)
$$


where $v \in \mathbb{R}^{m}$ is a nonlinear term to be designed. By substituting (8) in (7), we have the closed-loop system

$$
\begin{aligned}
\dot{\eta} & =a_{12}(\eta, \xi) \xi+\bar{d}_{1}(t, \eta, \xi) \\
\dot{\xi} & =v+d_{2}(t, \eta, \xi),
\end{aligned}
$$

with $\bar{d}_{1}(t, \eta, \xi)=d_{1}(t, \eta, \xi)+a_{11}(\eta, \xi) \eta$.

We now rewrite this system as an exact convex form following the methodology in [28], which is based on a generalisation of the sector nonlinearity approach [17] for tensor-product models [29].

Let us briefly recall this approach. To this end, consider $p$ to be the number of different non-constant bounded terms in $a_{12}(\eta, \xi)$, denoted as $z_{j} \in$ $\left[z_{j}^{0}, z_{j}^{1}\right], j \in\{1,2, \ldots, p\}$, with $z_{j}=\sum_{i_{j}=0}^{1} w_{i_{j}}^{j} z_{j}^{i_{j}}, w_{0}^{j}=\left(z_{j}^{1}-z_{j}\right) /\left(z_{j}^{1}-z_{j}^{0}\right)$, $w_{1}^{j}=1-w_{0}^{j}$. Thus, taking into account that convex sums can be stacked together at the leftmost side of an expression, we have that

$$
a_{12}(\eta, \xi)=\sum_{i_{1}=0}^{1} \sum_{i_{2}=0}^{1} \cdots \sum_{i_{p}=0}^{1} w_{i_{1}}^{1} w_{i_{2}}^{2} \cdots w_{i_{p}}^{p} A_{i_{1} i_{2} \cdots i_{p}}^{12} \equiv A_{w}^{12}
$$

with constant matrices $A_{i_{1} i_{2} \cdots i_{p}}^{12}=\left.a_{12}(\eta, \xi)\right|_{w_{i_{1}}^{1}=w_{i_{2}}^{2}=\cdots w_{i_{p}}^{p}=1}$. This results in the following equivalent regular convex model:

$$
\dot{x}=A_{w} x+B v+d(t, x), \quad x(0)=x_{0},
$$

with

$$
A_{w}=\left[\begin{array}{cc}
0 & A_{w}^{12} \\
0 & 0
\end{array}\right], \quad B=\left[\begin{array}{c}
0 \\
I_{m}
\end{array}\right], \quad d=\left[\begin{array}{l}
\bar{d}_{1} \\
d_{2}
\end{array}\right], \quad x=\left[\begin{array}{l}
\eta \\
\xi
\end{array}\right] .
$$

Remark 1. Notice that $a_{12}(\eta, \xi)$ is exactly $A_{w}^{12}$, the former is the nonlinear original expression while the latter stands for a rewriting of $a_{12}(\eta, \xi)$ as a convex sum of constant matrices $A_{i_{1} i_{2} \cdots i_{p}}^{12}$. This is a usual technique in the $L P V$ framework. Stability analysis of nonlinear systems via convex embedding in a polytope relies on the vertex systems, i.e., on each $A_{i_{1} i_{2} \cdots i_{p}}^{12}$ in the present case.

\subsection{Non-asymptotic stability and implicit Lyapunov functions}

We recall now the basics on finite- and fixed-time stability as well as how implicit Lyapunov functions can be used to guarantee them. 
Definition 1 ([30]). If the origin of (10) is an equilibrium point, it is said to be globally finite-time stable if it satisfies Lyapunov stability conditions and there exists a locally bounded function $T\left(x_{0}\right): \mathbb{R}^{n} \backslash\{0\} \rightarrow \mathbb{R}_{+}$, such that $\lim _{t \rightarrow T\left(x_{0}\right)} x\left(t, x_{0}\right)=0 \forall x_{0} \in \mathbb{R}^{n} \backslash\{0\}$. The function $T\left(x_{0}\right)$ is called a settlingtime function.

Definition 2 ([1]). If the origin of (10) is globally finite-time stable and $T\left(x_{0}\right)$ is bounded independently of the initial conditions (i.e. $\exists T_{M} \in \mathbb{R}_{+}$: $\left.T\left(x_{0}\right) \leq T_{M}, \forall x_{0} \in \mathbb{R}^{n}\right)$, then it is said to be globally fixed-time stable.

The proposed control design is based on the ILF approach, for which finite-time and fixed-time stability theorems are introduced below for the following differential inclusion

$$
\dot{x} \in F(t, x), \quad x(0)=x_{0},
$$

where the set-valued function $F: \mathbb{R}_{+} \times \mathbb{R}^{n} \rightrightarrows \mathbb{R}^{n}$ is compact, convex-valued and upper semi-continuous [31].

Lemma 1. [Finite-time stability [11]] The origin of (11) is globally finitetime stable if the next conditions are fulfilled:

C1) $\exists Q: \mathbb{R}_{+} \times \mathbb{R}^{n} \rightarrow \mathbb{R}$ continuously differentiable outside the origin;

C2) $\exists V \in \mathbb{R}_{+}: Q(V, x)=0, \forall x \in \mathbb{R}^{n} \backslash\{0\}$;

C3) let $\Omega=\left\{(V, x) \in \mathbb{R}_{+} \times \mathbb{R}^{n}: Q(V, x)=0\right\}$, and

$$
\lim _{\substack{x \rightarrow 0 \\(V, x) \in \Omega}} V=0, \lim _{\substack{V \rightarrow 0^{+} \\(V, x) \in \Omega}}\|x\|=0, \lim _{\substack{\|x\| \rightarrow \infty \\(V, x) \in \Omega}} V=+\infty
$$

C4) $\frac{\partial Q(V, x)}{\partial V}<0, \forall(V, x) \in \mathbb{R}_{+} \times \mathbb{R}^{n} \backslash\{0\}$;

$C 5)$ for some $c \in \mathbb{R}_{+}$and $\mu \in(0,1]$, the following is satisfied for $(V, x) \in \Omega$

$$
\sup _{t \in \mathbb{R}_{+}, y \in F(t, x)} \frac{\partial Q(V, x)}{\partial x} y \leq c V^{1-\mu} \frac{\partial Q(V, x)}{\partial V} .
$$

Moreover, the settling-time function estimate is $T\left(x_{0}\right) \leq \frac{V_{0}^{\mu}}{c \mu}$, for $V_{0} \in$ $\mathbb{R}_{+}: Q\left(V_{0}, x_{0}\right)=0$. 
Conditions C1)-C4) guarantee the existence and uniqueness of a smooth positive definite and radially unbounded Lyapunov function $V$ such that $Q(V, x)=0$ for all $x \in \mathbb{R}^{n}$, whilst condition C5) guarantees the time derivative of the Lyapunov function to be negative definite along the trajectories since from the implicit function theorem [32], $\frac{\partial V}{\partial x}=-\left[\frac{\partial Q}{\partial V}\right]^{-1} \frac{\partial Q}{\partial x}$, which implies the estimate of the time derivative $\dot{V}(x) \leq-c V^{1-\mu}$ and the aforementioned settling-time function.

Lemma 2. [Fixed-time stability [13]] The system (11) is globally fixed-time stable if there exist functions $Q_{1}$ and $Q_{2}$ that satisfy conditions C1)-C4) of Lemma 1 as well as the conditions:

C6) $Q_{1}(1, x)=Q_{2}(1, x)$;

C7) let $c_{1} \in \mathbb{R}_{+}$and $\mu \in(0,1]$, then $\forall V \in[0,1)$ and $x \in \mathbb{R}^{n} \backslash\{0\}$ such that $Q_{1}(V, x)=0$, it is satisfied

$$
\sup _{t \in \mathbb{R}_{+}, y \in F(t, x)} \frac{\partial Q_{1}(V, x)}{\partial x} y \leq c_{1} V^{1-\mu} \frac{\partial Q_{1}(V, x)}{\partial V} ;
$$

C8) let $c_{2} \in \mathbb{R}_{+}$and $\nu \in \mathbb{R}_{+}$, then $\forall V \geq 1$ and $x \in \mathbb{R}^{n} \backslash\{0\}$ such that $Q_{2}(V, x)=0$, it is satisfied

$$
\sup _{t \in \mathbb{R}_{+}, y \in F(t, x)} \frac{\partial Q_{2}(V, x)}{\partial x} y \leq c_{2} V^{1+\nu} \frac{\partial Q_{2}(V, x)}{\partial V} .
$$

Moreover, an estimation of the global settling-time function is $T\left(x_{0}\right) \leq$ $\frac{1}{c_{1} \mu}+\frac{1}{c_{2} \nu}$.

\section{Main results}

\subsection{Finite-time stabilization}

Consider the ILF

$$
Q(V, x)=x^{T} D_{r}\left(V^{-1}\right) P D_{r}\left(V^{-1}\right) x-1,
$$

where $V \in \mathbb{R}_{+}, P=P^{T} \in \mathbb{R}^{n \times n}>0$, and $D_{r}(\lambda), \lambda \in \mathbb{R}_{+}$has the form

$$
D_{r}(\lambda)=\left[\begin{array}{cc}
\lambda^{r_{1}} I_{n-m} & 0 \\
0 & \lambda^{r_{2}} I_{m}
\end{array}\right]
$$


with $r_{i}=1+(2-i) \mu, i=1,2$, and $0<\mu \leq 1$.

Notice that for $\mu=0$, the equation $Q(V, x)$ gives $V(x)=\sqrt{x^{T} P x}$ and for $\mu=1$, the ILF considered in [10] is recovered. Denote $H_{\mu}=\left[\begin{array}{cc}r_{1} I_{n-m} & 0 \\ 0 & r_{2} I_{m}\end{array}\right]$. In the sequel, adding the term $(*)$ to inline expressions denotes the transpose of the previous terms, i.e., $A+(*)=A+A^{T}$.

For the finite-time stabilization, consider the control law (8) with the nonlinear variable gain term

$$
v(V, x)=V^{1-\mu} K_{w} D_{r}\left(V^{-1}\right) x, \quad V: Q(V, x)=0,
$$

where $K_{w}=\sum_{i_{1}=0}^{1} \sum_{i_{2}=0}^{1} \cdots \sum_{i_{p}=0}^{1} w_{i_{1}}^{1} w_{i_{2}}^{2} \cdots w_{i_{p}}^{p} K_{i_{1} i_{2} \cdots i_{p}}$ and the design gains $K_{i_{1} i_{2} \cdots i_{p}}$ are provided in the following result:

Theorem 1. The trajectories of the disturbed system (10) under the control law (8) with (14), reach the origin in a finite time given by

$$
T\left(x_{0}\right) \leq \frac{V_{0}^{\mu}}{(1-\beta) \mu}, \quad Q\left(V_{0}, x_{0}\right)=0,
$$

if the following system of $L M I$

$$
\begin{gathered}
\left(A_{i_{1} \cdots i_{p}} X+B Y_{i_{1} \cdots i_{p}}+H_{\mu} X\right)+(*)+R<0 \\
X H_{\mu}+H_{\mu} X>0, \quad X>0
\end{gathered}
$$

is feasible for some $X \in \mathbb{R}^{n \times n}, Y_{i_{1} \cdots i_{p}} \in \mathbb{R}^{m \times n}$, a fixed $R \in \mathbb{R}^{n \times n}>0$, $K_{i_{1} \cdots i_{p}}=Y_{i_{1} \cdots i_{p}} X^{-1}, P=X^{-1}$, and disturbances satisfying the inequality

$$
\Upsilon^{T} R^{-1} \Upsilon \leq \beta V^{-2 \mu} x^{T} D_{r}\left(V^{-1}\right)\left(H_{\mu} P+P H_{\mu}\right) D_{r}\left(V^{-1}\right) x,
$$

with $\Upsilon=D_{r}\left(V^{-1}\right) d, \beta \in(0,1)$ and $V$ such that $Q(V, x)=0$.

Proof. Clearly, the ILF in (12) is continuously differentiable for all $(V, x) \in$ $\mathbb{R}_{+} \times \mathbb{R}^{n}$, and for any $x$ there exists a solution $V$ such that $Q(V, x)=0$, satisfying conditions $\mathrm{C} 1$ ) and $\mathrm{C} 2$ ) from Lemma 1. For condition C3), it is easy to show that the following chain of inequalities

$$
\frac{\lambda_{\min }(P)\|x\|^{2}}{\max \left\{V^{2+2(n-1) \mu}, V^{2}\right\}} \leq Q(V, x)+1 \leq \frac{\lambda_{\max }(P)\|x\|^{2}}{\min \left\{V^{2+2(n-1) \mu}, V^{2}\right\}}
$$

holds for all $(V, x) \in \mathbb{R}_{+} \times \mathbb{R}^{n}$, and for $Q(V, x)=0$, therefore the condition C3) holds. 
Thus, condition C4) of Lemma 1 also holds, since

$$
\begin{aligned}
\frac{\partial Q}{\partial V} & =-x^{T}\left[\begin{array}{cc}
r_{1} V^{-r_{1}-1} I_{n-m} & 0 \\
0 & r_{2} V^{-r_{2}-1} I_{m}
\end{array}\right] P D_{r}\left(V^{-1}\right) x-(*) \\
& =-V^{-1} x^{T} D_{r}\left(V^{-1}\right)\left[\begin{array}{cc}
r_{1} I_{n-m} & 0 \\
0 & r_{2} I_{m}
\end{array}\right] P D_{r}\left(V^{-1}\right) x-(*) \\
& =-V^{-1} x^{T} D_{r}\left(V^{-1}\right)\left(H_{\mu} P+P H_{\mu}\right) D_{r}\left(V^{-1}\right) x ;
\end{aligned}
$$

then, $\frac{\partial Q}{\partial V}<0$ is implied by $H_{\mu} P+P H_{\mu}>0$, and therefore condition C4) is verified.

Finally, by taking into account that $D_{r}\left(V^{-1}\right) A_{w} D_{r}^{-1}\left(V^{-1}\right)=V^{-\mu} A_{w}$ and $D_{r}\left(V^{-1}\right) B v=V^{-\mu} B K_{w} D_{r}\left(V^{-1}\right) x$, we have

$$
\begin{aligned}
\frac{\partial Q}{\partial x} \dot{x}= & x^{T} D_{r}\left(V^{-1}\right) P D_{r}\left(V^{-1}\right)\left(A_{w} x+B v+d\right)+(*) \\
= & V^{-\mu} x^{T} D_{r}\left(V^{-1}\right)\left(P A_{w}+P B K_{w}\right) D_{r}\left(V^{-1}\right) x+(*) \\
& +x^{T} D_{r}\left(V^{-1}\right) P D_{r}\left(V^{-1}\right) d+(*) \\
= & s^{T} W s+V^{\mu} d^{T} D_{r}\left(V^{-1}\right) R^{-1} D_{r}\left(V^{-1}\right) d \\
& -V^{-\mu} x^{T} D_{r}\left(V^{-1}\right)\left(H_{\mu} P+P H_{\mu}\right) D_{r}\left(V^{-1}\right) x
\end{aligned}
$$

with

$$
\begin{gathered}
s=\left[\begin{array}{c}
D_{r}\left(V^{-1}\right) x \\
D_{r}\left(V^{-1}\right) d
\end{array}\right], \\
W=\left[\begin{array}{cc}
V^{-\mu}\left(P\left(A_{w}+B K_{w}\right)+H_{\mu} P\right)+(*) & P \\
P & -V^{\mu} R^{-1}
\end{array}\right],
\end{gathered}
$$

for some matrix $R \in \mathbb{R}^{n \times n}, R>0$.

Considering $X=P^{-1}$ and applying Schur complement, $W<0$ iff

$$
\begin{aligned}
& V^{-\mu}\left(\left(A_{w} X+B K_{w} X+H_{\mu} X\right)+(*)+R\right) \\
& =V^{-\mu} \sum_{i_{1}=0}^{1} \sum_{i_{2}=0}^{1} \cdots \sum_{i_{p}=0}^{1} w_{i_{1}}^{1} w_{i_{2}}^{2} \cdots w_{i_{p}}^{p} \\
& \quad \times\left(\left(A_{i_{1} \cdots i_{p}} X+B K_{i_{1} \cdots i_{p}} X+H_{\mu} X\right)+(*)+R\right)<0 .
\end{aligned}
$$

Then, the set of LMIs

$$
A_{i_{1} \cdots i_{p}} X+X A_{i_{1} \cdots i_{p}}^{T}+B Y_{i_{1} \cdots i_{p}}+Y_{i_{1} \cdots i_{p}}^{T} B^{T}+H_{\mu} X+X H_{\mu}+R<0
$$


with $Y_{i_{1} \cdots i_{p}}=K_{i_{1} \cdots i_{p}} X$, and the fact that $\dot{V}=-\left[\frac{\partial Q}{\partial V}\right]^{-1} \frac{\partial Q}{\partial x} \dot{x}$, produces

$$
\begin{aligned}
\dot{V} \leq & -\left[\frac{\partial Q}{\partial V}\right]^{-1}\left(V^{\mu} d^{T} D_{r}\left(V^{-1}\right) R^{-1} D_{r}\left(V^{-1}\right) d\right. \\
& \left.-V^{-\mu} x^{T} D_{r}\left(V^{-1}\right)\left(H_{\mu} P+P H_{\mu}\right) D_{r}\left(V^{-1}\right) x\right) .
\end{aligned}
$$

For any continuous disturbance function $d$ satisfying (17) with $\beta \in(0,1)$, $V$ such that $Q(V, x)=0$, the estimate of the time derivative of $V$ is given by

$$
\begin{aligned}
\dot{V} & \leq\left[\frac{\partial Q}{\partial V}\right]^{-1} \frac{(1-\beta) x^{T} D_{r}\left(V^{-1}\right)\left(H_{\mu} P+P H_{\mu}\right) D_{r}\left(V^{-1}\right) x}{V^{\mu}} \\
& =-(1-\beta) V^{1-\mu},
\end{aligned}
$$

and therefore, condition C5) holds. The settling-time function estimate can be calculated by integration of the previous inequality, and is given in (15).

Remark 2. The reason to consider $a_{11}(\eta, \xi)$ as a perturbation in order to obtain the specific structure in $A_{w}$ can now be seen in the proof above, as otherwise the manipulations related with the dilation operator $D_{r}\left(V^{-1}\right)$ cannot be performed.

Remark 3. Notice that in order to satisfy restriction (17), the magnitude of the disturbances and the value of $\beta$ are directly related. This implies that the bound of the settling time is governed by the disturbance function. Therefore, stability conditions in [14], where nonlinearities are considered as exogenous disturbances, are more restrictive than ones obtained in this paper and produce larger settling times.

\subsection{Fixed-time stabilization}

For the fixed-time stabilization problem described in Lemma 2, consider the functions

$$
\begin{aligned}
& Q_{\mu}(V, x)=x^{T} D_{r_{\mu}}\left(V^{-1}\right) P D_{r_{\mu}}\left(V^{-1}\right) x-1, \\
& Q_{\nu}(V, x)=x^{T} D_{r_{\nu}}\left(V^{-1}\right) P D_{r_{\nu}}\left(V^{-1}\right) x-1
\end{aligned}
$$

where $V \in \mathbb{R}_{+}, P=P^{T} \in \mathbb{R}^{n \times n}>0$,

$$
D_{r_{\mu}}(\lambda)=\left[\begin{array}{cc}
\lambda^{1+\mu} I_{n-m} & 0 \\
0 & \lambda I_{m}
\end{array}\right], D_{r_{\nu}}(\lambda)=\left[\begin{array}{cc}
\lambda I_{n-m} & 0 \\
0 & \lambda^{1+\nu} I_{m}
\end{array}\right],
$$


$0<\mu \leq 1, \nu \in \mathbb{R}_{+}$. Denote $H_{\mu}=\operatorname{diag}\left\{(1+\mu) I_{n-m}, I_{m}\right\}$ and $H_{\nu}=$ $\operatorname{diag}\left\{I_{n-m},(1+\nu) I_{m}\right\}$.

Consider the control law (8) with

$$
v(V, x)=\left\{\begin{aligned}
V^{1-\mu} K_{w} D_{r_{\mu}}\left(V^{-1}\right) x, & x^{T} P x<1 \\
V^{1+2 \nu} K_{w} D_{r_{\nu}}\left(V^{-1}\right) x, & x^{T} P x \geq 1
\end{aligned}\right.
$$

with $K_{w}=\sum_{i_{1}=0}^{1} \sum_{i_{2}=0}^{1} \cdots \sum_{i_{p}=0}^{1} w_{i_{1}}^{1} w_{i_{2}}^{2} \cdots w_{i_{p}}^{p} K_{i_{1} i_{2} \cdots i_{p}}$ and $V$ defined as

$$
V:\left\{\begin{array}{lll}
Q_{\mu}(V, x)=0 & \text { for } \quad x^{T} P x<1 \\
Q_{\nu}(V, x)=0 & \text { for } \quad x^{T} P x \geq 1
\end{array}\right.
$$

Theorem 2. The closed-loop system (10) under the control law (8) with (19), (20), is fixed-time stable with the settling time estimate

$$
T\left(x_{0}\right) \leq \frac{1}{\left(\alpha_{\mu}-\beta_{\mu}\right) \mu}+\frac{1}{\left(\alpha_{\nu}-\beta_{\nu}\right) \nu},
$$

if the system of $L M I$

$$
\begin{aligned}
& \left(A_{i_{1} \cdots i_{p}} X+B Y_{i_{1} \cdots i_{p}}+\alpha_{\mu} H_{\mu} X\right)+(*)+R_{\mu} \leq 0, \\
& \left(A_{i_{1} \cdots i_{p}} X+B Y_{i_{1} \cdots i_{p}}+\alpha_{\nu} H_{\nu} X\right)+(*)+R_{\nu} \leq 0, \\
& X H_{\mu}+H_{\mu} X>0, \quad X H_{\nu}+H_{\nu} X>0, \quad X>0
\end{aligned}
$$

is feasible for some $X \in \mathbb{R}^{n \times n}, Y_{i_{1} \cdots i_{p}} \in \mathbb{R}^{m \times n}, \alpha_{\mu}, \alpha_{\nu} \in \mathbb{R}_{+}$, some fixed matrices $R_{\mu}, R_{\nu} \in \mathbb{R}^{n \times n}>0, K_{i_{1} \cdots i_{p}}=Y_{i_{1} \cdots i_{p}} X^{-1}, P=X^{-1}$, and disturbances satisfying the inequalities

$$
\begin{aligned}
& \Upsilon_{\mu}^{T} R_{\mu}^{-1} \Upsilon_{\mu} \leq \beta_{\mu} V^{-2 \mu} x^{T} D_{r_{\mu}}\left(V^{-1}\right)\left(H_{\mu} P+P H_{\mu}\right) D_{r_{\mu}}\left(V^{-1}\right) x \\
& \Upsilon_{\nu}^{T} R_{\nu}^{-1} \Upsilon_{\nu} \leq \beta_{\nu} V^{2 \nu} x^{T} D_{r_{\nu}}\left(V^{-1}\right)\left(H_{\nu} P+P H_{\nu}\right) D_{r_{\nu}}\left(V^{-1}\right) x
\end{aligned}
$$

if $x^{T} P x \leq 1$ and $x^{T} P x \geq 1$, respectively, for $\Upsilon_{\mu}=D_{r_{\mu}}\left(V^{-1}\right) d, \Upsilon_{\nu}=$ $D_{r_{\nu}}\left(V^{-1}\right) d, \beta_{\mu} \in\left[0, \alpha_{\mu}\right)$, and $\beta_{\nu} \in\left[0, \alpha_{\nu}\right)$.

Proof. Fixed-time stability conditions C1)-C4) follow a similar outline as for Theorem 1 since the functions defined in (18) preserve the properties of (12).

Hence

$$
\begin{aligned}
& \frac{\partial Q_{\mu}}{\partial V}=-V^{-1} x^{T} D_{r_{\mu}}\left(V^{-1}\right)\left(H_{\mu} P+P H_{\mu}\right) D_{r_{\mu}}\left(V^{-1}\right) x \\
& \frac{\partial Q_{\nu}}{\partial V}=-V^{-1} x^{T} D_{r_{\nu}}\left(V^{-1}\right)\left(H_{\nu} P+P H_{\nu}\right) D_{r_{\nu}}\left(V^{-1}\right) x
\end{aligned}
$$


such that condition C4) holds for $H_{\mu} P+P H_{\mu}>0$ and $H_{\nu} P+P H_{\nu}>0$.

Obviously, condition C6) of Lemma 2, i.e. $Q_{\mu}(1, x)=Q_{\nu}(1, x)$ is satisfied by (18). Notice that $x^{T} P x \leq 1 \Rightarrow V(x) \leq 1$ and $x^{T} P x \geq 1 \Rightarrow V(x) \geq 1$.

Applying similar arguments as for the proof of Theorem 1, for condition C7) we have

$$
\begin{aligned}
\frac{\partial Q_{\mu}}{\partial x} \dot{x}= & s^{T} W_{\mu} s+V^{\mu} d^{T} D_{r_{\mu}}\left(V^{-1}\right) R_{\mu}^{-1} D_{r_{\mu}}\left(V^{-1}\right) d \\
& -\alpha_{\mu} V^{-\mu} x^{T} D_{r_{\mu}}\left(V^{-1}\right)\left(H_{\mu} P+P H_{\mu}\right) D_{r_{\mu}}\left(V^{-1}\right) x
\end{aligned}
$$

with

$$
\begin{gathered}
s=\left[\begin{array}{c}
D_{r_{\mu}}\left(V^{-1}\right) x \\
D_{r_{\mu}}\left(V^{-1}\right) d
\end{array}\right], \\
W_{\mu}=\left[\begin{array}{cc}
V^{-\mu}\left(P\left(A_{w}+B K_{w}\right)+\alpha_{\mu} H_{\mu} P\right)+(*) & P \\
P & -V^{\mu} R_{\mu}^{-1}
\end{array}\right],
\end{gathered}
$$

and finally, the first line of LMIs in (22) with $P=X^{-1}, K_{i_{1} \cdots i_{p}}=Y_{i_{1} \cdots i_{p}} X^{-1}$ and the disturbance restriction in (23), guarantee

$$
\dot{V} \leq-\left(\alpha_{\mu}-\beta \mu\right) V^{1-\mu}
$$

for $V(x) \leq 1$.

In the case of the function $Q_{\nu}(V, x)$, i.e. $V(x) \geq 1$, taking into account that $D_{r_{\nu}}\left(V^{-1}\right) A_{w} D_{r_{\nu}}^{-1}\left(V^{-1}\right)=V^{\nu} A_{w}$ and $D_{r_{\nu}}\left(V^{-1}\right) B v=V^{\nu} B K_{w} D_{r_{\nu}}\left(V^{-1}\right) x$, we obtain

$$
\begin{aligned}
\frac{\partial Q_{\nu}}{\partial x} \dot{x}= & s^{T} W_{\nu} s+V^{-\nu} d^{T} D_{r_{\nu}}\left(V^{-1}\right) R_{\nu}^{-1} D_{r_{\nu}}\left(V^{-1}\right) d \\
& -\alpha_{\nu} V^{\nu} x^{T} D_{r_{\nu}}\left(V^{-1}\right)\left(H_{\nu} P+P H_{\nu}\right) D_{r_{\nu}}\left(V^{-1}\right) x
\end{aligned}
$$

for

$$
W_{\nu}=\left[\begin{array}{cc}
V^{\nu}\left(P\left(A_{w}+B K_{w}\right)+\alpha_{\nu} H_{\nu} P\right)+(*) & P \\
P & -V^{-\nu} R_{\nu}^{-1}
\end{array}\right] .
$$

Hence, the second line of LMIs in (22) guarantee

$$
\dot{V}=-\left[\frac{\partial Q_{\nu}}{\partial V}\right]^{-1} \frac{\partial Q_{\nu}}{\partial x}\left(A_{w} x+B v+d\right) \leq-\left(\alpha_{\nu}-\beta_{\nu}\right) V^{1+\nu}
$$

and therefore, condition C8) holds. The settling-time function can be calculated by integration of the inequalities corresponding to the time derivative of $V$, giving the estimate in (21). 
It is helpful to remark that the convergence time of the fixed-time stable system does not depend on the initial condition. Moreover, parameters $\alpha_{\mu}$ and $\alpha_{\nu}$ were introduced in order to tune the convergence time of the closedloop system.

\subsection{Parametric uncertainties}

The proposed methodology can be generalized in order to deal with a more realistic scenario when the system is in the presence of parametric uncertainties. To do so, consider the following generalization of the closedloop system in (9):

$$
\begin{aligned}
\dot{\eta} & =a_{12}(\eta, \xi, \theta) \xi+\bar{d}_{1}(t, \eta, \xi, \theta) \\
\dot{\xi} & =v+d_{2}(t, \eta, \xi, \theta)
\end{aligned}
$$

with $\bar{d}_{1}$ and $d_{2}$ preserving the same meaning as before, though $a_{j k}, j, k \in$ $\{1,2\}$ are allowed to include time-varying bounded parametric uncertainties $\theta(t)$.

Based on the methodology presented in section 2, the previous system could be rewritten as the following regular convex model

$$
\dot{x}=\mathbf{A}_{\mathbf{w}_{\mathbf{i}} \omega_{\mathbf{j}}} x+B v+d(t, x, \theta), \quad x(0)=x_{0},
$$

with

$$
\begin{aligned}
\mathbf{A}_{\mathbf{w}_{\mathbf{i}} \omega_{\mathbf{j}}}= & {\left[\begin{array}{cc}
0 & \mathbf{A}_{\mathbf{\mathbf { w } _ { \mathbf { i } }} \omega_{\mathbf{j}}}^{\mathbf{1 2}} \\
0 & 0
\end{array}\right], \quad B=\left[\begin{array}{c}
0 \\
I_{m}
\end{array}\right], \quad d=\left[\begin{array}{l}
\bar{d}_{1} \\
d_{2}
\end{array}\right], \quad x=\left[\begin{array}{l}
\eta \\
\xi
\end{array}\right] } \\
& \mathbf{A}_{\mathbf{w}_{\mathbf{i}} \omega_{\mathbf{j}}}^{\mathbf{1 2}}=\sum_{\mathbf{i} \in \mathbb{B}^{p}} \sum_{\mathbf{j} \in \mathbb{B}^{\rho}} \mathbf{w}_{\mathbf{i}} \omega_{\mathbf{j}} \mathbf{A}_{\mathbf{i j}}{ }^{12} \equiv a_{12}(\eta, \xi, \theta),
\end{aligned}
$$

for $\mathbb{B}=\{0,1\}, \mathbf{i}=\left(i_{1}, \cdots, i_{p}\right), \mathbf{j}=\left(j_{i}, \cdots, j_{\rho}\right), \mathbf{A}_{\mathbf{i j}}{ }^{12}=\left.a_{12}(\eta, \xi, \theta)\right|_{\mathbf{w}_{\mathbf{i}} \omega_{\mathbf{j}}=1}$ and certain nonlinearities grouped in functions $\mathbf{w}_{\mathbf{i}}(\eta, \xi)=w_{i_{1}}^{1} w_{i_{2}}^{2} \cdots w_{i_{p}}^{p}$, whilst uncertain ones are grouped in functions $\omega_{\mathbf{j}}(\theta)=w_{j_{1}}^{1} w_{j_{2}}^{2} \cdots w_{j_{\rho}}^{\rho}$. These nonlinear terms are split in order to synthesize a completely certain control law by excluding the uncertainties, i.e. for the finite-time stabilization case, the nonlinear variable gain term of the control law is given by

$$
v(V, x)=V^{1-\mu} \mathbf{K}_{\mathbf{w}_{\mathbf{i}}} D_{r}\left(V^{-1}\right) x, \quad V: Q(V, x)=0,
$$

where $\mathbf{K}_{\mathbf{w}_{\mathbf{i}}}=\sum_{i_{1}=0}^{1} \cdots \sum_{i_{p}=0}^{1} w_{i_{1}}^{1} \cdots w_{i_{p}}^{p} K_{i_{1} \cdots i_{p}}$ with constant gain matrices $K_{i_{1} i_{2} \cdots i_{p}}$ to be designed. Notice that $\mathbf{K}_{\mathbf{w}_{\mathbf{i}}}$ is completely certain since the uncertain terms grouped in $\omega_{\mathbf{j}}$ are excluded from it. 
Corollary 1. The origin of the uncertain system (24), (26) is finite-time stable if

$$
\begin{gathered}
\left(\mathbf{A}_{\mathbf{i j}} X+B \mathbf{Y}_{\mathbf{i}}+H_{\mu} X\right)+(*)+R<0 \\
X H_{\mu}+H_{\mu} X>0, \quad X>0
\end{gathered}
$$

is feasible for some $X \in \mathbb{R}^{n \times n}$ and $\mathbf{Y}_{\mathbf{i}}=Y_{i_{1} \cdots i_{p}} \in \mathbb{R}^{m \times n}$ such that $K_{i_{1} \cdots i_{p}}=$ $Y_{i_{1} \cdots i_{p}} X^{-1}$, a fixed $R \in \mathbb{R}^{n \times n}>0$, and disturbances satisfying the inequality in $(17)$.

Proof. Recalling the ILF in (12) which clearly satisfies conditions C1), C2), and C3), whilst condition C4) is still implied by $H_{\mu} P+P H_{\mu}>0$, we have for C5)

$$
\begin{aligned}
\frac{\partial Q}{\partial x} \dot{x}= & x^{T} D_{r}\left(V^{-1}\right) P D_{r}\left(V^{-1}\right)\left(\mathbf{A}_{\mathbf{w}_{\mathbf{i}} \omega_{\mathbf{j}}} x+B v+d\right)+(*) \\
= & s^{T} W s+V^{\mu} d^{T} D_{r}\left(V^{-1}\right) R^{-1} D_{r}\left(V^{-1}\right) d \\
& -V^{-\mu} x^{T} D_{r}\left(V^{-1}\right)\left(H_{\mu} P+P H_{\mu}\right) D_{r}\left(V^{-1}\right) x
\end{aligned}
$$

with

$$
\begin{gathered}
s=\left[\begin{array}{c}
D_{r}\left(V^{-1}\right) x \\
D_{r}\left(V^{-1}\right) d
\end{array}\right], \\
W=\left[\begin{array}{cc}
V^{-\mu}\left(P\left(\mathbf{A}_{\mathbf{w}_{\mathrm{i}} \omega_{\mathbf{j}}}+B K_{w}\right)+H_{\mu} P\right)+(*) & P \\
P & -V^{\mu} R^{-1}
\end{array}\right] .
\end{gathered}
$$

By following a similar outline as for Theorem 1, the set of LMIs

$$
\mathbf{A}_{\mathbf{i j}} X+X \mathbf{A}_{\mathbf{i j}}^{T}+B \mathbf{Y}_{\mathbf{i}}+\mathbf{Y}_{\mathbf{i}}^{T} B^{T}+H_{\mu} X+X H_{\mu}+R<0
$$

and the restriction in (17) produce

$$
\dot{V} \leq-(1-\beta) V^{1-\mu} .
$$

It is easy to see that the settling-time function estimate is also given in (15).

Corollary 2. The origin of the uncertain closed-loop system (24) under the control law

$$
v(V, x)=\left\{\begin{array}{cc}
V^{1-\mu} \mathbf{K}_{\mathbf{w}_{\mathbf{i}}} D_{r_{\mu}}\left(V^{-1}\right) x, & x^{T} P x<1 \\
V^{1+2 \nu} \mathbf{K}_{\mathbf{w}_{\mathbf{i}}} D_{r_{\nu}}\left(V^{-1}\right) x, & x^{T} P x \geq 1
\end{array}\right.
$$


where $\mathbf{K}_{\mathbf{w}_{\mathbf{i}}}=\sum_{i_{1}=0}^{1} \cdots \sum_{i_{p}=0}^{1} w_{i_{1}}^{1} \cdots w_{i_{p}}^{p} K_{i_{1} \cdots i_{p}}$ and $V$ is defined as in (20), is fixed-time stable with the settling time estimate in (21) if the following set of $L M I$

$$
\begin{gathered}
\left(\mathbf{A}_{\mathbf{i j}} X+B \mathbf{Y}_{\mathbf{i}}+\alpha_{\mu} H_{\mu} X\right)+(*)+R_{\mu} \leq 0, \\
\left(\mathbf{A}_{\mathbf{i j}} X+B \mathbf{Y}_{\mathbf{i}}+\alpha_{\nu} H_{\nu} X\right)+(*)+R_{\nu} \leq 0, \\
X H_{\mu}+H_{\mu} X>0, \quad X H_{\nu}+H_{\nu} X>0, \quad X>0
\end{gathered}
$$

is feasible for some $X \in \mathbb{R}^{n \times n}, \mathbf{Y}_{\mathbf{i}}=Y_{i_{1} \cdots i_{p}} \in \mathbb{R}^{m \times n}, \alpha_{\mu}, \alpha_{\nu} \in \mathbb{R}_{+}$, some fixed matrices $R_{\mu}, R_{\nu} \in \mathbb{R}^{n \times n}>0, K_{i_{1} \cdots i_{p}}=Y_{i_{1} \cdots i_{p}} X^{-1}$, and disturbances satisfying (23).

Proof. It follows a similar outline as for Theorem 2 in combination with Corollary 1.

Remark 4. The proposed methodology is also able to deal with nonlinear systems in the form

$$
\dot{x}=\left[\begin{array}{ccccc}
0 & A_{12}(x) & 0 & \cdots & 0 \\
0 & 0 & A_{23}(x) & \cdots & 0 \\
\vdots & \vdots & \vdots & \ddots & \vdots \\
0 & 0 & 0 & \cdots & A_{n-m n}(x) \\
0 & 0 & 0 & \cdots & 0
\end{array}\right] x+\left[\begin{array}{c}
0 \\
0 \\
\vdots \\
0 \\
I_{m}
\end{array}\right] v+d(t, x)
$$

with only slight modifications to the dilation matrix $D_{r}\left(V^{-1}\right)$ and $H_{\mu}$ (see [14] for details) and produces exactly the same results as in Theorems 1 and 2, and Corollaries 1 and 2.

\subsection{Control algorithm implementation}

As it can be seen in (14) and (19), the practical implementation of the control law (8) requires $V(x)$ to be known. This can be realized by finding the solution $V$ of the equation $Q(V, x)=0$ analytically, or in-line using the actual value of the state vector. A simple algorithm based on the bisection method for the localization of the control parameter $V_{i}$ at each sampling time instance $t_{i}$ (digital implementation), is shown in Table 1 [11], where parameters $V_{\min }$ and $V_{0}$ define the initial lower and higher possible values of $V$, respectively. For finite numerical precision of the digital implementation, $V_{\min }$ (or $V_{0}$ ) must not be selected arbitrary small (or big). 
Table 1: Bisection method [11].

\begin{tabular}{||l||}
\hline Algorithm for the selection of $V_{i}$ \\
\hline \hline INITIALIZATION: $a=V_{\min } ; b=V_{0} ;$ \\
METHOD: \\
If $x_{i}^{T} D_{r}\left(b^{-1}\right) P D_{r}\left(b^{-1}\right) x_{i}>1$ then \\
$\quad a=b ; b=2 b ;$ \\
elseif $x_{i}^{T} D_{r}\left(a^{-1}\right) P D_{r}\left(a^{-1}\right) x_{i}<1$ then \\
$\quad b=a ; a=\max \left\{\frac{a}{2}, V_{\min }\right\} ;$ \\
else $c=\frac{a+b}{2} ;$ \\
If $x_{i}^{T} D_{r}\left(c^{-1}\right) P D_{r}\left(c^{-1}\right) x_{i}<1$ then \\
$\quad b=c ;$ \\
$\quad$ else $a=\max \left\{V_{\min }, c\right\} ;$ \\
endif \\
endif \\
$V_{i}=b ;$
\end{tabular}

The method consists in the localization of the value $V\left(x_{i}\right)=V_{i}$ at each sampling time instance $t_{i}$ such that the equation $Q\left(V_{i}, x_{i}\right)=0$ is fulfilled with $x_{i} \in \mathbb{R}^{n}$ as some given vector. If METHOD section of this algorithm is applied many times at the sampling instant $t_{i}$ for the same $x_{i}$ (a loop containing METHOD), then the algorithm provides: i) a calculation of the unique positive root of the equation $Q\left(V, x_{i}\right)=0$, i.e. $V\left(x_{i}\right)=V_{i} \in[a, b]$; ii) improvement of the calculation by means of the bisection method, i.e. $|a-b| \rightarrow 0$. Such a loop allows us to calculate $V_{i}$ with higher precision, nevertheless, if the computational power is very restricted, the METHOD section of the algorithm may be realized only once or a few times at each sampling time instance.

Moreover, for $\mu \in(0,1)$, the control scheme (14) is a continuous function of the state $x$. Nevertheless, if $\mu=1$ then the control is continuous only outside the origin and globally bounded such that $x^{T} \operatorname{Dr}\left(V^{-1}\right) \operatorname{PDr}\left(V^{-1}\right) x=$ 1 implies $\left\|\operatorname{Dr}\left(V^{-1}\right) x\right\|^{2} \leq \frac{1}{\lambda_{\min }(P)}$ and $\|v\|^{2} \leq\|K\|^{2} \cdot\left\|\operatorname{Dr}\left(V^{-1}\right) x\right\|^{2} \leq \frac{\|K\|}{\lambda_{\min }(P)}$. The LMI

$$
\left[\begin{array}{cc}
X & Y^{T} \\
Y & v_{0}^{2} I_{m}
\end{array}\right] \geq 0
$$


can be solved together with (16) in order to restrict the control magnitude by $\|v\| \leq v_{0}$.

Corollary 3. If conditions in Theorem 1 hold and $v=v\left(V_{i}, x\right)$ with $V_{i} \in \mathbb{R}_{+}$ obtained from the aforementioned digital implementation, then the ellipsoid

$$
\Pi\left(V_{i}, X^{-1}\right):=\left\{x \in \mathbb{R}^{n}: x^{T}\left(D_{r}\left(V_{i}\right) X D_{r}\left(V_{i}\right)\right)^{-1} x \leq 1\right\}
$$

is a positively invariant set of the closed-loop system (10), (8), i.e. $x\left(t_{i}\right) \in$ $\Pi\left(V_{i}, X^{-1}\right) \Rightarrow x(t) \in \Pi\left(V_{i}, X^{-1}\right), t>t_{i}$, where $t_{i}$ is the sampling time instance for the realization of the ILF control algorithm.

Proof. From the first line of the LMI system in (16) we have

$$
D_{r}\left(V_{i}^{-1}\right)\left(P\left(A_{i_{1} \cdots i_{p}}+H_{\mu}+B K_{i_{1} \cdots i_{p}}\right)+(*)+P R P\right) D_{r}\left(V_{i}^{-1}\right)<0 .
$$

Let $\mathcal{P}=D_{r}\left(V_{i}^{-1}\right) P D_{r}\left(V_{i}^{-1}\right)$, since $D_{r}^{-1}\left(V_{i}^{-1}\right) A_{i_{1} \cdots i_{p}} D_{r}\left(V_{i}^{-1}\right)=V^{\mu} A_{i_{1} \cdots i_{p}}$, $D_{r}^{-1}\left(V_{i}^{-1}\right) B=V_{i} B$, and $D_{r}^{-1}\left(V_{i}^{-1}\right) H_{\mu} D_{r}\left(V_{i}^{-1}\right)=H_{\mu}$, it follows that

$$
\mathcal{P}\left(A_{i_{1} \cdots i_{p}}+B \bar{K}_{i_{1} \cdots i_{p}}+\frac{1}{V_{i}^{\mu}} H_{\mu}\right)+(*)+\frac{1}{V_{i}^{\mu}} \mathcal{P} D_{r}\left(V_{i}\right) R D_{r}\left(V_{i}\right) \mathcal{P}<0,
$$

where $\bar{K}_{i_{1} \cdots i_{p}}=V_{i}^{1-\mu} K_{i_{1} \cdots i_{p}} D_{r}\left(V_{i}^{-1}\right)$ and therefore, $v\left(V_{i}, x\right)=\bar{K}_{i_{1} \cdots i_{p}} x$.

To conclude the proof, consider the quadratic Lyapunov function $\mathcal{V}=$ $x^{T} \mathcal{P} x$, then

$$
\begin{aligned}
\dot{\mathcal{V}}= & {\left[\begin{array}{l}
x \\
d
\end{array}\right]^{T} W_{i}\left[\begin{array}{l}
x \\
d
\end{array}\right]-\frac{1}{V_{i}^{\mu}} x^{T}\left(H_{\mu} \mathcal{P}+\mathcal{P} H_{\mu}\right) x } \\
& +V_{i}^{\mu} d^{T} D_{r}\left(V_{i}^{-1}\right) R^{-1} D_{r}\left(V_{i}^{-1}\right) d \\
\leq & -\frac{1-\beta}{V_{i}^{\mu}} x^{T}\left(H_{\mu} \mathcal{P}+\mathcal{P} H_{\mu}\right) x, \forall x \in \mathbb{R}^{n}: Q\left(V_{i}, x\right)=0,
\end{aligned}
$$

because $W_{i}=\left[\begin{array}{cc}\Psi_{i_{1} \cdots i_{p}} & P \\ P & -V_{i}^{\mu}\left(D_{r}\left(V_{i}\right) R D_{r}\left(V_{i}\right)\right)^{-1}\end{array}\right]$ with

$$
\Psi_{i_{1} \cdots i_{p}}=\mathcal{P}\left(A_{i_{1} \cdots i_{p}}+B \bar{K}_{i_{1} \cdots i_{p}}+\frac{H_{\mu}}{V_{i}^{\mu}}\right)+(*)
$$

is guarantee to be negative definite by Schur complement of the inequality above and the condition in (17). Therefore, the ellipsoid $\Pi\left(V_{i}, P\right)$ is strictly positively invariant set of the closed-loop system (10) with $v=v\left(V_{i}, x\right)$. 


\section{Numerical results}

Example 1: The second-order nonlinear system (1) from the motivation example is now resumed. The substitution of the control law $u=-x_{1}^{2}-x_{2}+v$ in (1) with $v$ as the nonlinear term to be designed via the ILF method produces

$$
\dot{x}=\left[\begin{array}{cc}
0 & \theta+0.5+2 \operatorname{sinc}\left(x_{2}\right) \\
0 & 0
\end{array}\right] x+\left[\begin{array}{l}
0 \\
1
\end{array}\right] v+\underbrace{\left[\begin{array}{c}
0 \\
f_{m}(t, x)
\end{array}\right]}_{d(t, x)},
$$

where $v$ is designed via the proposed nonlinear methodology dealing with $d(t, x)$ as exogenous disturbances.

The methodology proposed in this paper begins by rewriting the nonlinear representation above into the form (10), for that purpose, it is clear that for $\left|x_{2}\right| \leq 2$, the certain nonlinear term $z=2 \operatorname{sinc}\left(x_{2}\right) \in[0.9,2]=\left[z^{0}, z^{1}\right]$ leads to $w_{0}^{1}=(2-z) / 1.1$ and $w_{1}^{1}=1-w_{0}^{1}$, while the uncertain one $\bar{z}=\theta \in$ $[-0.2,0.2]=\left[\bar{z}^{0}, \bar{z}^{1}\right]$ leads to $\omega_{0}^{1}=(0.2-\bar{z}) / 0.4$ and $\omega_{1}^{1}=1-\omega_{0}^{1}$, such that

$$
\dot{x}=\sum_{i_{1}=0}^{1} \sum_{j_{1}=0}^{1} w_{i_{1}}^{1} \omega_{j_{1}}^{1}\left[\begin{array}{cc}
0 & 0.5+z^{i_{1}}+\bar{z}^{j_{1}} \\
0 & 0
\end{array}\right] x+\left[\begin{array}{l}
0 \\
1
\end{array}\right] v+d(t, x) .
$$

In order to implement $u$ with $v=V^{1-\mu} \sum_{i_{1}=0}^{1} w_{i_{1}}^{1} K_{i_{1}} D_{r}\left(V^{-1}\right) x$ (only depending on the certain nonlinear terms), LMIs in theorem 1 were found feasible and restriction in (17) were satisfied for $\mu=1, R=I_{2}$, and $\beta=0.18$, together with (31) for $v_{0}=10$, obtaining

$$
\begin{aligned}
& P=\left[\begin{array}{ll}
5.6502 & 2.4803 \\
2.4803 & 1.2524
\end{array}\right], \\
& K_{0}=\left[\begin{array}{ll}
-19.0655 & -9.9136
\end{array}\right], \quad K_{1}=\left[\begin{array}{ll}
-16.5226 & -10.1025
\end{array}\right] \text {, }
\end{aligned}
$$

such that the settling-time function estimate provides $T\left(x_{0}\right) \leq \frac{V_{0}}{0.82}=0.5488$.

In contrast, methodology in [13] is not able to deal with this system for the reasons argued in the motivation example, while LMI conditions in [14] were found feasible for the given parameters $\mu=1, R=I_{2}$, and $v_{0}=10$, but the disturbance restriction therein only mets for values of $\beta$ bigger than 0.5 , which provides a settling-time estimate $T\left(x_{0}\right) \leq 0.9$.

For simulation purposes a step size $h=0.01$ was taken into account such that the algorithm in Table 1 found a numerical solution $V_{i}$ of the equation 


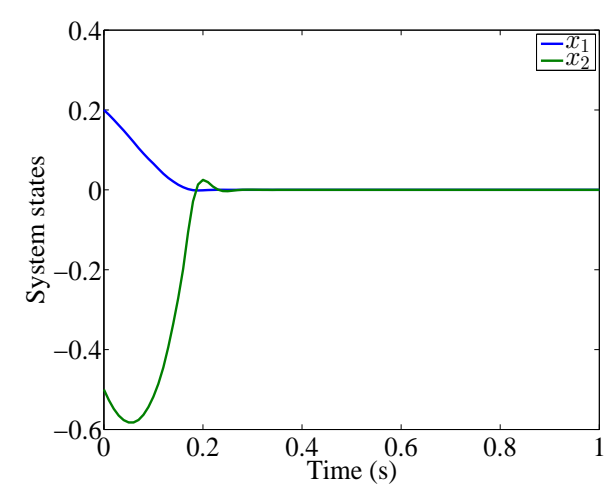

(a) The proposed ILF control

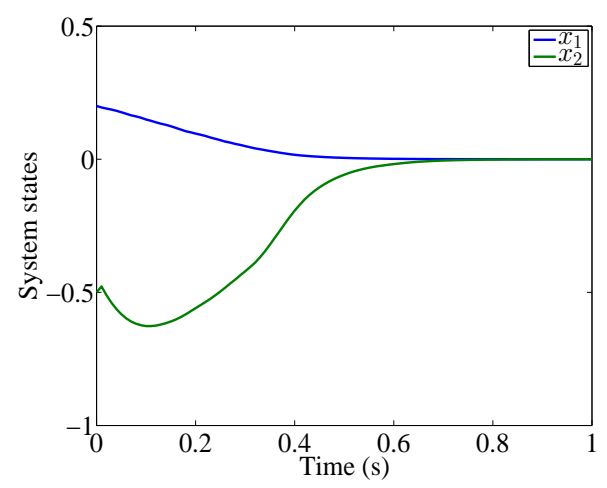

(b) The ILF control in [14]

Figure 1: Time evolution of the states.

$Q(V, x)=0$ at the time instant $t_{i}$. Fig. 1 (a) shows the evolution of the trajectories of system (1) under the designed ILF control (26) and Fig. 1 (b) shows the simulation results for the ILF control proposed in [14], both for initial conditions $x(0)=\left[\begin{array}{ll}0.2 & -0.5\end{array}\right]^{T}$ and an exogenous disturbance $f_{m}=\sin \left(10 x_{1}\right)$.

Example 2: Consider a nonlinear system from [33]:

$$
\dot{\chi}=\left[\begin{array}{c}
0 \\
0 \\
\chi_{2}+\chi_{3}^{2} \\
\chi_{2}-\chi_{2} e^{\chi_{3}}-\chi_{4}
\end{array}\right]+\left[\begin{array}{ll}
1 & 0 \\
0 & 1 \\
0 & 0 \\
1 & 1
\end{array}\right] u \text {. }
$$

With the change of coordinates $\eta=\left[\begin{array}{ll}\eta_{1} & \eta_{2}\end{array}\right]^{T}=\left[\begin{array}{ll}\chi_{4}-\chi_{1}-\chi_{2} & \chi_{3}\end{array}\right]^{T}$, $\xi=\left[\begin{array}{ll}\xi_{1} & \xi_{2}\end{array}\right]^{T}=\left[\begin{array}{ll}\chi_{2} & \chi_{1}+\chi_{2}\end{array}\right]^{T}$, the system (33) can be described by the regular form

$$
\begin{aligned}
& \dot{\eta}=\underbrace{\left[\begin{array}{cc}
-1 & 0 \\
0 & \eta_{2}
\end{array}\right]}_{a_{11}(\eta, \xi)} \eta+\underbrace{\left[\begin{array}{cc}
1-e^{\eta_{2}} & -1 \\
1 & 0
\end{array}\right]}_{a_{12}(\eta, \xi)} \xi \\
& \dot{\xi}=\underbrace{\left[\begin{array}{ll}
0 & 1 \\
1 & 1
\end{array}\right]}_{b} u,
\end{aligned}
$$


where $z^{0}=0<e^{\eta_{2}} \leq z^{1}$ and for design purposes, $a_{11}(\eta, \xi) \eta$ is considered as an exogenous disturbance. Notice that $b$ is invertible and $\mathfrak{T}\left(\chi_{1}, \chi_{2}, \chi_{3}, \chi_{4}\right) \mapsto$ $\left(\eta_{1}, \eta_{2}, \xi_{1}, \xi_{2}\right)$ is a diffeomorphism since the Jacobian matrix of $\mathfrak{T}$ is nonsingular.

In order to obtain the equivalent regular convex model (10), it is clear that $z=e^{\eta_{2}} \in\left[0, z^{1}\right]$ leads to $w_{0}^{1}=\left(z^{1}-z\right) / z^{1}$ and $w_{1}^{1}=1-w_{0}^{1}$, such that

$$
\dot{x}=\sum_{i_{1}=0}^{1} w_{i_{1}}^{1}\left[\begin{array}{cccc}
0 & 0 & 1-z^{i_{1}} & -1 \\
0 & 0 & 1 & 0 \\
0 & 0 & 0 & 0 \\
0 & 0 & 0 & 0
\end{array}\right] x+\left[\begin{array}{ll}
0 & 0 \\
0 & 0 \\
1 & 0 \\
0 & 1
\end{array}\right] v+d(t, x)
$$

Solving LMIs in (16) for $\mu=1, R=\operatorname{diag}\{0.25,0.25,0.25,0.25\}, z^{1}=3$, and $v_{0}=10$, we obtain

$$
\begin{aligned}
P & =\left[\begin{array}{cccc}
1.4854 & 1.3506 & 0.1125 & -0.5578 \\
1.3506 & 20.9906 & 3.4530 & -0.4862 \\
0.1125 & 3.4530 & 0.8529 & -0.0377 \\
-0.5578 & -0.4862 & -0.0377 & 0.2407
\end{array}\right], \\
K_{0} & =\left[\begin{array}{cccc}
-3.4846 & -36.1241 & -8.3064 & 1.3086 \\
7.6267 & -4.2403 & -0.4680 & -4.0161
\end{array}\right], \\
K_{1} & =\left[\begin{array}{llll}
0.6843 & -32.6525 & -7.9369 & -0.2682 \\
8.2726 & 13.8285 & 0.2878 & -4.2027
\end{array}\right] .
\end{aligned}
$$

The algorithm in Table 1 was implemented for a step size $h=0.01$ with $V_{\min }=0.1$ and for initial conditions $x(0)=\left[\begin{array}{llll}0.5 & -0.5 & -0.5 & 0.5\end{array}\right]^{T}$. Fig. 2 (a) shows the evolution of the states of system (33) whilst the control inputs are depicted on Fig. 2 (b), validating the performance of the designed ILF control.

\section{Conclusions}

This paper presents an ILF control design for robust stabilization of multiinput nonlinear systems that allows handling non-asymptotic (finite-time or fixed-time) convergence. The design permits to consider several nonlinearities and parametric uncertainties as a part of the nominal model instead of exogenous disturbances, that is reflected in less restrictive robust stability conditions and smaller settling times than the existing literature concerning 


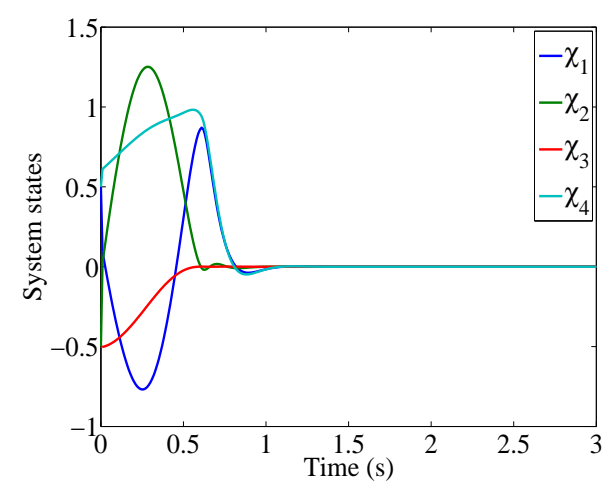

(a) Time evolution of the states

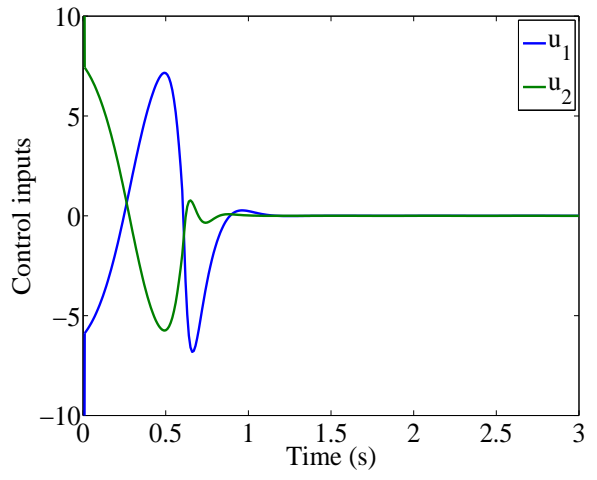

(b) Control inputs

Figure 2: Simulation results.

the linear case. Design conditions are formulated as LMIs by mimicking the linear case by means of the use of convex representations, which provides constructive tuning of the controller gains.

\section{Acknowledgment}

The authors gratefully acknowledge the financial support of CONACyT project 282013 for CVU 631139, the CONACYT sabbatical fellowship for CVU 202355, PAPIIT-UNAM IN 115419, the Government of Russian Federation (Grant 08-08), the Ministry of Science and Higher Education of Russian Federation, passport of goszadanie no. 2019-0898, and ITSON projects PFCE 2018-19 and PROFAPI 2019-0002.

\section{References}

[1] A. Polyakov, Nonlinear feedback design for fixed-time stabilization of linear control systems, IEEE Transactions on Automatic Control 57 (8) (2012) 2106-2110.

[2] C. Edwards, S. Spurgeon, Sliding mode control: Theory and applications, Taylor and Francis, London, England, 1998.

[3] A. Levant, Higher-order sliding modes, differentiation and output feedback control, International Journal of Control 76 (9-10) (2003) 924-941. 
[4] U. Perez-Ventura, L. Fridman, When is it reasonable to implement the discontinuous sliding-mode controllers instead of the continuous ones? Frequency domain criteria, International Journal of Robust and Nonlinear Control 29 (3) (2019) 810-828.

[5] A. Levant, Homogeneity approach to high-order sliding mode design, Automatica 41 (5) (2005) 823-830.

[6] J. Moreno, M. Osorio, Strict Lyapunov functions for the super-twisting algorithm, IEEE Transactions on Automatic Control 57 (4) (2012) 10351040 .

[7] S. Ding, A. Levant, S. Li, Simple homogeneous sliding-mode controller, Automatica 67 (2016) 22-32.

[8] E. Cruz-Zavala, J. Moreno, Homogeneous high order sliding mode design: A Lyapunov approach, Automatica 80 (2017) 232-238.

[9] V. Korobov, A solution of the problem of synthesis using a controllability function, Doklady Academii Nauk SSSR 248 (5) (1979) 1051-1055.

[10] J. Adamy, A. Fleming, Soft variable-structure controls: a survey, Automatica 40 (11) (2004) 1821-1844.

[11] A. Polyakov, D. Efimov, W. Perruquetti, Finite-time stabilization using implicit Lyapunov function technique, in: 9th Symposium of Nonlinear Control Systems, The International Federation of Automatic Control, Toulouse, France, 2013, pp. 140-145.

[12] A. Polyakov, D. Efimov, W. Perruquetti, Sliding mode control design for MIMO systems: Implicit Lyapunov function approach, in: European Control Conference, Strasbourg, France, 2014, pp. 2612-2617.

[13] A. Polyakov, D. Efimov, W. Perruquetti, Finite-time and fixed-time stabilization: Implicit Lyapunov function approach, Automatica 51 (2015) $332-340$.

[14] A. Polyakov, D. Efimov, W. Perruquetti, Robust stabilization of MIMO systems in finite/fixed time, International Journal of Robust and Nonlinear Control 26 (1) (2016) 69-90. 
[15] J. Li, H. Wang, D. Niemann, K. Tanaka, Synthesis of gain-scheduled controller for a class of LPV systems, in: Proceedings of the 38th IEEE Conference on Decision and Control, Phoenix, USA, 1999, pp. 23142319 .

[16] P. Park, D. Choi, LPV controller design for the nonlinear RTAC system, International Journal of Robust and Nonlinear Control 11 (14) (2001) 1343-1363.

[17] T. Taniguchi, K. Tanaka, H. Wang, Model construction, rule reduction, and robust compensation for generalized form of Takagi-Sugeno fuzzy systems, IEEE Transactions on Fuzzy Systems 9 (4) (2001) 525-538.

[18] K. Tanaka, H. Wang, Fuzzy Control Systems Design and Analysis: A Linear Matrix Inequality Approach, John Wiley and Sons, New York, USA, 2001.

[19] A. Sala, C. Arino, Polynomial fuzzy models for nonlinear control: a Taylor series approach, IEEE Transactions on Fuzzy Systems 17 (6) (2009) 1284-1295.

[20] S. Boyd, L. El Ghaoui, E. Feron, V. Balakrishnan, Linear matrix inequalities in system and control theory, Vol. 15, Studies in Applied Mathematics, Philadelphia, USA, 1994.

[21] P. Gahinet, A. Nemirovsky, A. Laub, M. Chilali, LMI control toolbox, Math Works, Natick, USA, 1995.

[22] J. Sturm, Using SeDuMi 1.02, a MATLAB toolbox for optimization over symmetric cones, Optimization Methods and Software 11 (1-4) (1999) 625-653.

[23] C. Edwards, A practical method for the design of sliding mode controllers using linear matrix inequalities, Automatica 40 (10) (2004) 17611769 .

[24] H. Choi, K. Ro, LMI-based sliding-mode observer design method, Control Theory and Applications, IEE Proceedings 152 (1) (2005) 113-115.

[25] A. Tapia, R. Márquez, M. Bernal, J. Cortez, Sliding subspace design based on linear matrix inequalities, Kybernetika 50 (3) (2014) 436-449. 
[26] R. Márquez, A. Tapia, M. Bernal, L. Fridman, LMI-based second order sliding set design using reduced order of derivatives, International Journal of Robust and Nonlinear Control 25 (18) (2015) 3763-3779.

[27] A. Tapia, M. Bernal, L. Fridman, An LMI approach for second-order sliding set design using piecewise Lyapunov functions, Automatica 79 (2017) 61-64.

[28] A. Tapia, M. Bernal, L. Fridman, Nonlinear sliding mode control design: an LMI approach, Systems \& Control Letters 104 (2017) 38-44.

[29] C. Ariño, A. Sala, Relaxed LMI conditions for closed-loop fuzzy systems with tensor-product structure, Engineering Applications of Artificial Intelligence 20 (8) (2007) 1036-1046.

[30] Y. Orlov, Finite time stability and robust control synthesis of uncertain switched systems, SIAM Journal on Control and Optimization 43 (4) (2005) 1253-1271.

[31] A. Filippov, Differential Equations with Discontinuous Right-hand Sides, Kluwer Academic Publishers, Dordrecht, The Netherlands, 1988.

[32] R. Courant, F. John, Introduction to Calculus and Analysis (Vol. II/I), Springer-Verlag, Berlin, Germany, 1999.

[33] A. Isidori, Nonlinear Control Systems, 3rd Edition, Springer-Verlag, London, England, 1995. 